\title{
Pathologies of Modular Algebraic Surfaces
}

\section{Citation}

Mumford, David B. 1961. Pathologies of modular algebraic surfaces. American Journal of Mathematics 83(2): 339-342.

\section{Published Version}

doi:10.2307/2372959

\section{Permanent link}

http://nrs.harvard.edu/urn-3:HUL.InstRepos:3446005

\section{Terms of Use}

This article was downloaded from Harvard University's DASH repository, and is made available under the terms and conditions applicable to Other Posted Material, as set forth at http:// nrs.harvard.edu/urn-3:HUL.InstRepos:dash.current.terms-of-use\#LAA

\section{Share Your Story}

The Harvard community has made this article openly available.

Please share how this access benefits you. Submit a story.

Accessibility 


\title{
Pathologies of Modular Algebraic Surfaces
}

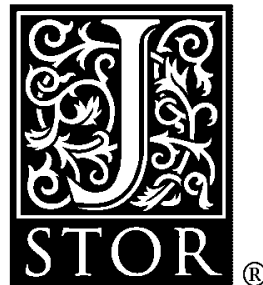

\author{
David Mumford
}

American Journal of Mathematics, Vol. 83, No. 2 (Apr., 1961), 339-342.

Stable URL:

http://links.jstor.org/sici?sici=0002-9327\%28196104\%2983\%3A2\%3C339\%3APOMAS\%3E2.0.CO\%3B2-X

American Journal of Mathematics is currently published by The Johns Hopkins University Press.

Your use of the JSTOR archive indicates your acceptance of JSTOR's Terms and Conditions of Use, available at http://www.jstor.org/about/terms.html. JSTOR's Terms and Conditions of Use provides, in part, that unless you have obtained prior permission, you may not download an entire issue of a journal or multiple copies of articles, and you may use content in the JSTOR archive only for your personal, non-commercial use.

Please contact the publisher regarding any further use of this work. Publisher contact information may be obtained at http://www.jstor.org/journals/jhup.html.

Each copy of any part of a JSTOR transmission must contain the same copyright notice that appears on the screen or printed page of such transmission.

JSTOR is an independent not-for-profit organization dedicated to creating and preserving a digital archive of scholarly journals. For more information regarding JSTOR, please contact support@jstor.org. 


\title{
PATHOLOGIES OF MODULAR ALGEBRAIC SURFACES.*
}

\author{
By Datid Mumford.
}

The purpose of this note is to present two counterexamples to conjectures about the geometry of algebraic surfaces, which are, on the contrary, true in characteristic zero. Thus if $F$ is a non-singular algebraic surface defined over an algebraically closed field $k$, one can form the vector spaces

$$
\begin{aligned}
& H_{0,1}=H^{1}\left(F, \Omega^{0}\right), \\
& H_{1,0}=H^{0}\left(F, \Omega^{1}\right),
\end{aligned}
$$

where $\Omega^{i}$ is the sheaf of regular $i$-forms. One also has the vector space

$$
\begin{aligned}
A_{0}= & \text { cotangent space to the Albanese Variety } \\
& A \text { of } F \text { at the origin } 0 .
\end{aligned}
$$

Among these vector spaces various maps may be defined regardless of the ground field. First, the canonical map

induces a homomorphism

$$
\phi: F \rightarrow A
$$

$$
\phi^{*}: A_{0} \rightarrow H_{1,0},
$$

which Igusa [4] has shown to be one-one. Secondly, if an embedding of $F$ in projective space is fixed, and hence a canonical element $h$ in $H^{1}\left(F, \Omega^{1}\right)$, then cup product and Serre duality induces a pairing

$$
\text { If : } H_{0,1} \times H_{1,0} \rightarrow k \text {. }
$$

(See Kodaira [6] and Serre [9]).

Now in the classical case, the usual constructive existence proof of the Albanese variety $A$ shows immediately that $\phi^{*}$ is onto (see Weil [12]); while the famous topological results of Lefschetz on the embeddings of varieties in projective space (see Wallace [11], and Kodaira [6]), allow one to conclude that the pairing $\mathscr{H}$ is non-degenerate. One deduces the "fundamental equalities" of Italian surface theory:

$$
\operatorname{dim} A_{0}=\operatorname{dim} H_{1,0}=\operatorname{dim} H_{0,1},
$$

\footnotetext{
* Received January 4, 1961.
} 
proven first by Poincaré by his method of normal functions in 1910, (Poincaré [8]). A corollary of $\phi^{*}$ being onto, combined with the easy proof (valid in all characteristics-see Koizumi [7]) that regular differentials on an Abelian variety are closed, shows that the differentials in $H_{1,0}$ are all closed. This has been generalized by Hodge, and has led ultimately to the result: analytic regular differentials on a Kähler manifold are closed (see Weil [12]).

The question is what extends to the modular, or non-zero characteristic case. Igusa showed, first of all, that $\phi^{*}$ is not always onto [5]. Serre showed next that the pairing $\mathscr{H}$ is not always non-degenerate, since it can happen that $H_{1,0}=(0)$, while $H_{0,1} \neq(0)$, (see [10]). There remain the questions:

(a) Are all differentials in $H_{1,0}$ closed?

(b) Does the pairing $\mathscr{\Psi}$ have the property that if $x$ in $H_{1,0}$ is such that $\dddot{\Psi}(x, y)=0$ for all $y$ in $H_{0,1}$, then $x=0$ ?

The answer to both is no.

I.

To answer (a), we prove (assuming the characteristic $\neq 0$ ):

THEOREM. Let $F$ be a non-singular algebraic surface, $\omega$ any simple differential on $F$ (i.e. 1-form) ; then there exists a non-singular surface $F^{*}$ and a regular map $\phi: F^{*} \rightarrow F$ which is separable and algebraic, such that $\phi^{*}(\omega)$ is regular on $F^{*}$.

Proof. Note first that it is enough to prove:

(\#) For all $P$ in $F$, there exists an open $U$ containing $P$, a surface $F^{*}$ and a regular map $\phi: F^{*} \rightarrow F$ separable and algebraic such that $\phi^{-1}(U)$ is non-singular, and $\phi^{*}(\omega)$ is regular on $\phi^{-1}(U)$.

If this is proven, then we can find a finite set $U_{i}^{*}$ of open sets covering $F$ and of surfaces $F_{i}{ }^{*}$, and maps $\phi_{i}: F_{i}{ }^{*} \rightarrow F$ with the above properties. Then let $F^{*}$ be some non-singular model of the function field of any compositum of $k\left(F_{i}^{*}\right)$ (compatible with the identification of the common subfields $k(F)$ ), that dominates the models $F_{i}^{*}$ (such exists by Abhyankar [1] and Zariski [13]). Let $\phi$ be the map from $F^{*}$ to $F$. Then for all $P$ in $F^{*}$, say $\phi(P)$ in $U_{i}$. Then $\phi_{i}{ }^{*}(\omega)$ is regular at $\phi_{i}{ }^{-1}(\phi(P))$, hence $\phi^{*}(\omega)$ is regular at $P$.

But in fact it is enough to prove:

(\#\#) Same as (\#) except for differentials $\omega=A d x, x$ a uniformizing parameter at $P$. 
For if at $P, \omega=A d x+B d y$, then pick $F_{1}{ }^{*}$ that does the trick for $A d x$ and then $F_{2}{ }^{*}$ that does the trick for $B d y$, and take a suitable non-singularization of their join as in (\#).

But now if $\omega=\left(A_{0} / A_{1}\right) d x, A_{0}$ and $A_{1}$ regular at $P$, and $A_{1}=0$ at $P$, consider the extension It follows

$$
Z^{P}+\left(A_{1}{ }^{P} \cdot Z\right)+(x)=0 \text {, where } p \text { is the characteristic. }
$$

$$
d x=-A_{1}{ }^{P} d Z .
$$

Now in the normalization $F^{*}$ of $F$ in this field extension, there is a unique point $P^{*}$ above $P$. Moreover, it is simple since if $x, y$ are uniformizing parameters at $P$, then $Z, y$ are uniformizing parameters at $P^{*}$. Finally,

$$
\omega=\left(A_{0} / A_{1}\right) d x=A_{0} A_{1}^{p-1}\left(d x / A_{1}^{p}\right)=-A_{0} A_{1}^{p-1} d Z .
$$

Hence $\omega$ is regular at $P^{*}$, hence in an open set about $P^{*}$. QED

COROLLARY. There exist algebraic surfaces, non-singular and with a simple regular differential $\omega$ that is not closed.

Proof. Take in the above theorem $F=P^{2}$, the projective plane, and $\omega=x d y$. Then the differential $\phi^{*}(\omega)$ on the covering $F^{*}$ given by the theorem satisfies the corollary. One must merely note that $d \phi^{*}(\omega)=\phi^{*}(d \omega)$ $\neq 0$ as the covering is separable. QED

II.

To answer (b), consider the famous Enriques surface $E$, which is, in any characteristic, the normalization of the sextic surface $E_{0}$ in $P^{3}$ :

$$
0=x^{2} y^{2} z^{2}+x^{2} y^{2}+x^{2} z^{2}+y^{2} z^{2}+x y z f_{2}(x, y, z),
$$

where $f_{2}$ is a general polynomial of second degree. Its normalization is nonsingular and, regardless of characteristic, can be constructed as the join of the graphs of the following set of maps:

$$
\begin{aligned}
& \phi_{1}: E_{0} \rightarrow P^{1}, \text { given by } x y / z, \\
& \phi_{2}: E_{0} \rightarrow P^{1}, \text { given by } x z / y, \\
& \phi_{3}: E_{0} \rightarrow P^{1}, \text { given by } y z / x,
\end{aligned}
$$

as the reader may with some pains verify. It follows that the surface $E$ in characteristic 2 is a specialization of $E$ in characteristic 0 , hence has the same $p_{a}$ (see Hironaka [3]), which has long been known to be 0 (for a modern 
treatment, see Artin's thesis [2]). On the other hand, the classical theory of adjoint surfaces tells us that canonical divisors on $E$, as divisors on $E_{0}$, must be cut out by $(6-4)=2$ nd order forms in $P^{3}$ passing through the double lines (see Zariski [14]); in this case, we have a tetrahedron of double lines and such are contained in no quadric. Hence $p_{g}=0$, hence $H^{0,1}=(0)$.

Now in the remarkable case of characteristic 2 , it is also the case that $H^{1,0} \neq(0)$. In fact, let $t$ be a coordinate on $P^{1}$; then :

$$
\phi_{1}^{*}(d t)=\phi_{2}^{*}(d t)=\phi_{3}^{*}(d t)=d(x y z)
$$

is immediately seen to be a regular differential on $E$.

HARVARD UNIVERSITY.

\section{REFERENCES.}

[1] S. Abhyankar, "Local uniformization of algebraic surfaces over ground fields of characteristic $p \neq 0$, , Annals of Mathematics, vol. 63 (1956), p. 491.

[2] M. Artin, On Enriques' Surfaces, Doctoral thesis, Harvard, 1960.

[3] H. Hironaka, "A note on algebraic geometry over ground rings," Illinois Journal of Mathematics, vol. 2 (1958), p. 355.

[4] J. I. Igusa, "A fundamental inequality in the Theory of Picard varieties," Proceedings of the National Academy of Sciences, USA, vol. 41 (1955), p. 317.

[5] — " "On some problems in abstract algebraic geometry," ibid., vol. 41 (1955), p. 964.

[6] K. Kodaira and D. C. Spencer, "On a theorem of Lefschetz and the lemma of Enriques-Severi-Zariski," Proceedings of the National Academy of Sciences, $U S A$, vol. 39 (1953), p. 1273.

[7] S. Koizumi, "On differential forms of the first kind on algebraic varieties," Journal of the Mathematical Society of Japan, vol. 1 (1949), p. 273.

[8] H. Poincaré, "Sur les Courbes Tracee sur les Surfaces Algebriques," Annales de l'Ecole Normale Supérieure, vol. 27 (1910), p. -

[9] J. P. Serre, “Faisceaux Algebriques Coherents," Annals of Mathematics, vol. 61 (1955), p. 197.

[10] — " Sur la topologie des varieties algebriques en caracteristique $p$," Symposium on Algebraic Topology, Mexico, 1956.

[11] A. Wallace, Homology Theory on Algebraic Varieties, Pergamon Press, 1958.

[12] A. Weil, Varietes Kahleriennes, Paris, 1958.

[13] O. Zariski, "Simplified proof for the resolution of singularities of an algebraic surface," Annals of Mathematics, vol. 43 (1942), p. 583.

[14] - An Introduction to the Theory of Algebraic Surfaces, Mimeographed Lee ture Notes, Harvard, 1957-8. 